\title{
The Influence of Using a Static Diastolic Geometry in ECG Imaging
}

\author{
Job Stoks $^{1,2,3}$, Matthijs JM Cluitmans ${ }^{1}$, Ralf Peeters ${ }^{2}$, Paul GA Volders ${ }^{1}$ \\ ${ }^{1}$ Department of Cardiology, Maastricht University Medical Centre, Maastricht, the Netherlands \\ ${ }^{2}$ Data Science and Knowledge Engineering, Maastricht University, Maastricht, the Netherlands \\ ${ }^{3}$ BIOMED, Hasselt University, Hasselt, Belgium
}

\begin{abstract}
One of the common choices when performing electrocardiographic imaging (ECGI) is that the cardiac geometry is in a static, diastolic state. To test the influence of this approximation, we compared epicardial potential maps and isochrones during systolic and diastolic geometries in four patients. Zero-th order Tikhonov regularization was used to reconstruct ventricular epicardial potentials. A spatiotemporal estimation method was then used to determine the activation and recovery times from the reconstructed epicardial electrograms. Activation times (AT), recovery times $(R T)$ and electrogram correlation coefficients $(C C)$ were compared for both geometries. Furthermore, $C C$ and differences in $A T / R T$ were correlated against the linear movement and a substitute for rotational movement. Poor correlation was found between linear/rotational movement and reconstruction differences. Overall, agreement between epicardial potential maps and isochrones of both geometries was high when assessed quantitatively, but regional differences might occur for qualitative interpretation. These differences mostly occurred in areas of flat T-waves. This novel, more accurate quantification of the influence of assuming a diastolic geometry in ECGI may further help in interpreting ECGI measurements.
\end{abstract}

\section{Introduction}

Electrocardiographic imaging (ECGI) is a modality that noninvasively images electrical activation and recovery directly on the heart surface. ECGI reconstructs direct representations of electrical activity on the heart surface by combining extensive recordings from $\sim 200$ body-surface electrodes with a precise, patient-specific torso-heart geometry.[1] ECGI provides more detailed and localized information than the clinical electrocardiogram (ECG), and has previously been quantitatively validated by our group [2] and others. [1] However, due to the ill-posedness of the inverse problem and common approximations when reconstructing electrical activity at the heart surface, these epicardial potential maps and isochrones contain a certain inaccuracy. One of the common approximations when performing ECGI is that the cardiac geometry is in a mechanically static (non-moving) state, usually taken from the diastolic phase of the cardiac cycle.[1] To investigate and quantify influence of this approximation, we compared epicardial potential maps and isochrones during systolic and diastolic geometries for four patients without structural heart disease. The systolic and diastolic states are analyzed since they are the two most extreme phases of the mechanically moving heart. Quantitative differences between geometries, the locations of these differences and the consequences on the clinical interpretation of the inverse solution are evaluated.

\section{Methods}

\subsection{BSPM and geometry}

The study was approved by the local ethical committee and all patients gave written informed consent before the $\mathrm{CT}$ and body surface potential mapping (BSPM) took place. A helical electrocardiography (ECG)-gated CT scan was performed with intravenous iodine contrast medium and both a systolic and diastolic phase of the torso-heart geometry were reconstructed. Segmentation of the ventricular epicardium was performed in a fully automated manner (Philips Intellispace version 10) and manually validated afterwards. Geometries were subsequently reduced to an average of $2483 \pm 26$ nodes. The average node-to-node distance in one geometry was $3.8 \pm 0.3 \mathrm{~mm}$. Recordings were made using a 256-channel acquisition system of which $134 \pm 8$ were used on average, with a 2048-Hz sampling rate (BioSemi, Amsterdam, the Netherlands). For each subject, two separate sinus beats were analyzed.

\subsection{Preprocessing and reconstruction}

Before the inverse reconstruction was made, baseline drift and $50 \mathrm{~Hz}$ noise were removed from the BSPM. The STT-segment was filtered with a $2^{\text {nd }}$ order $40 \mathrm{~Hz}$ lowpass Butterworth filter. Subsequently, inverse reconstructions of epicardial potentials were performed on the basis of the potential-based formulation of ECGI [1]; we used an 
epicardium-only formulation [2]. Subsequently, the commonly used zero-th order Tikhonov regularization was used to reconstruct epicardial potentials. A spatiotemporal estimation method was then used to determine the activation and recovery times from the reconstructed epicardial electrograms.[3] This method takes advantage of the spatial relationship between neighboring nodes and their potentials, providing a more accurate inverse solution.[2] Activation times (ATs) and recovery times (RTs) are expressed relative to the first moment of epicardial activation.

\subsection{Comparison of inverse solutions}

To compare the inverse solution of diastolic and systolic geometries, electrograms of the diastolic geometry were compared to their nearest neighbors in the systolic geometry. The artificial plane closing the ventricles at the base (a method commonly used to obtain a completely closed surface as required for computational stability) was not taken into account for analysis. ATs, RTs and electrogram morphologies during the QRS-complex and STT-segment were compared between both geometries.

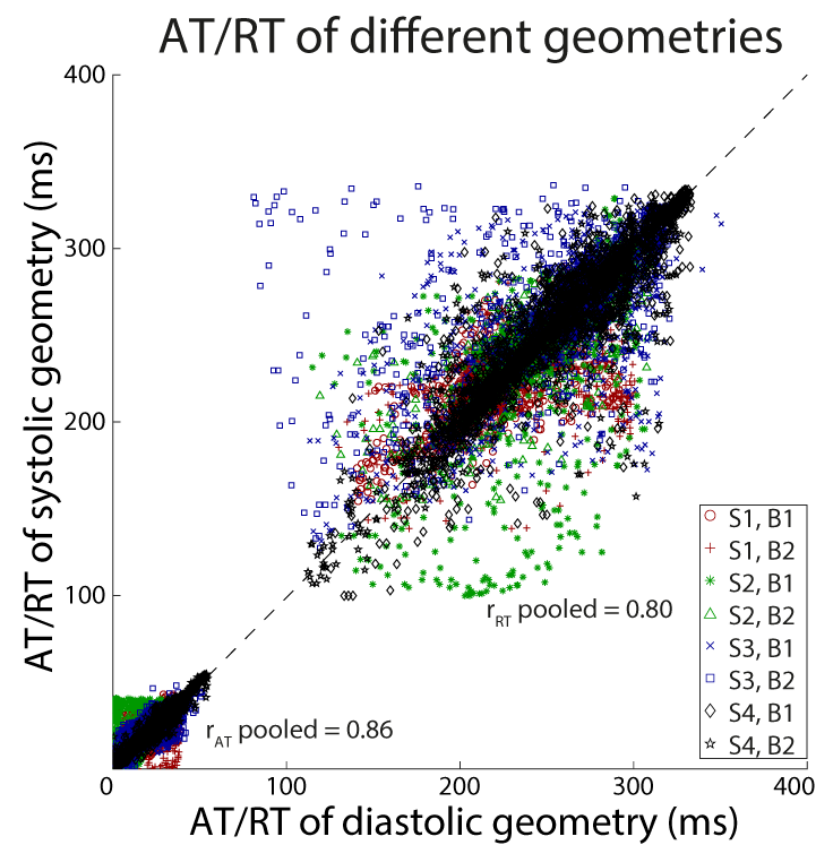

Figure 1: ATs and RTs of diastolic and systolic geometries. S: subject. B: beat.
These comparisons are expressed as correlation coefficients $\left(\mathrm{r}_{\mathrm{AT}}, \mathrm{r}_{\mathrm{RT}}, \mathrm{CC}_{\mathrm{QRS}}\right.$ and $\mathrm{CC}_{\mathrm{STT}}$, respectively). Furthermore, a qualitative comparison is made between RT maps, to investigate the consequences of these differences on clinical interpretation. Since the epicardial wall movement was not equal throughout the heart, we also relate these correlation coefficients to epicardial wall movement. Lastly, since the rotational movement of the heart is negatively correlated with the distance to the apex [4] but cannot be obtained from CT scans directly, we also assess the relation between the outcome measures and the distance to the apex.

\section{Results}

\subsection{Subject characteristics}

Four patients without structural heart disease were retrospectively included in the study. Patient characteristics are shown in Table 1. Two males and two females were included, with a mean age of $50 \pm 12$ years. Mean length and weight were $173 \pm 10 \mathrm{~cm}$ and $87 \pm 14 \mathrm{~kg}$, respectively. Mean left ventricular (LV) end diastolic volume (LVEDV), end systolic volume (LVESV) and ejection fraction (LVEF) retrieved from clinical MRI were $167 \pm 19 \mathrm{ml}, 59 \pm 11 \mathrm{ml}$ and $65 \pm 7 \%$, respectively.

\subsection{Differences in inverse solution}

Differences in epicardial potential maps and isochrones between diastolic and systolic geometry were addressed in terms of AT, RT, CC during activation and $\mathrm{CC}$ during repolarization. Results of $r_{A T}$ and $r_{R T}$ of pooled data are shown in Figure 1. Considerable differences were observed for different individuals. Overall, there was good correlation between depolarization times $\left(\mathrm{r}_{\mathrm{AT}}=0.78\right.$ (interquartile range (IQR) $0.75-0.89)$ ) and recovery times $\left(\mathrm{r}_{\mathrm{RT}}=0.74(0.62-0.91)\right)$ of different geometries. $\mathrm{CC}_{\mathrm{QRS}}$ and $\mathrm{CC}_{\text {STT }}$ were both 0.99 (0.95-1.00). Differences in RT seemed to occur mostly at the inferior side, see Figure 2. Even though the quantitative parameters show little difference, qualitative interpretation of systolic and diastolic maps may still differ considerably (as shown in the second column). Regions with high RT differences seemed to be coinciding with regions of large repolarization heterogeneities.

Table 1: Patient characteristics. LVESV: left ventricular end systolic volume, LVEDV: left ventricular end diastolic volume, LVEF: left ventricular ejection fraction. Parameters calculated with MRI.

\begin{tabular}{llllllll}
\hline ID & Gender & Age $(\mathrm{y})$ & Length $(\mathrm{cm})$ & Weight $(\mathrm{kg})$ & LVEDV $(\mathrm{ml})$ & LVESV $(\mathrm{ml})$ & LVEF $(\%)$ \\
\hline 1 & M & 35 & 173 & 78 & 193 & 71 & 63 \\
2 & $\mathrm{~F}$ & 57 & 170 & 108 & 166 & 49 & 71 \\
3 & $\mathrm{~F}$ & 63 & 162 & 82 & 147 & 65 & 56 \\
4 & $\mathrm{M}$ & 45 & 185 & 80 & 160 & 50 & 69 \\
\hline
\end{tabular}




\subsection{Linear movement}

Overall, the median linear movement of the different nodes on the heart averaged over the individuals was 4.0 (IQR 2.6-6.1) $\mathrm{mm}$. The artificial basal nodes, which are not taken into account in further analyses, moved 7.7 (5.9-9.5) $\mathrm{mm}$, in contrast to 3.6 (2.4-5.1) for the non-artificial nodes. Figure 2, column 4 shows the absolute linear movement of the heart. Correlations of absolute linear movement vs. AT and $\mathrm{RT}$ differences, $\mathrm{CC}_{\mathrm{QRS}}$ and $\mathrm{CC}_{\mathrm{STT}}$ were also addressed. Overall, these correlations were low $(\mathrm{r}=0.08(0.06-0.13)$, 0.05 (0.03-0.08), 0.06 (0.01-0.17) and 0.09 (0.07-0.12), respectively), meaning that linear movement of nodes and differences in inverse solution seem uncorrelated.

\subsection{Distance from apex}

Correlation of distance to the apex (as surrogate for rotational movement) vs. AT/RT differences and $\mathrm{CC}_{\mathrm{QRS}}$ and $\mathrm{CC}_{\text {STT }}$ was addressed Overall, these correlations were low ( $\mathrm{r}=0.14$ (IQR 0.11-0.31), 0.06 (0.01-0.14), 0.04 (0.030.11 ) and 0.12 (0.04-0.17), respectively), meaning that longitudinal distance to the apex of nodes (and thus rotational movement) and differences in epicardial potential maps and isochrones seem uncorrelated.

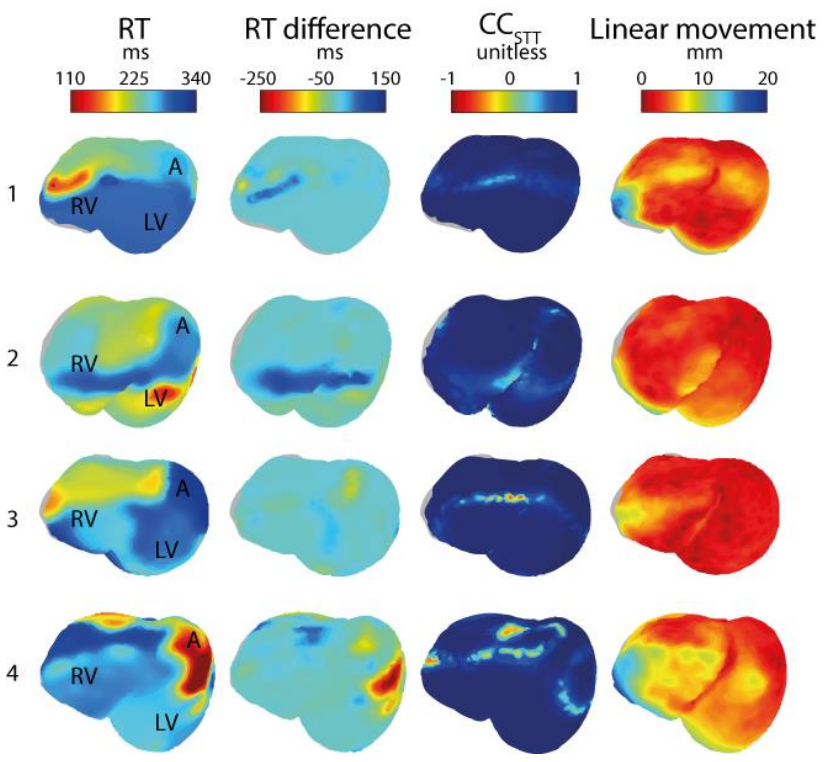

Figure 2: Diastolic geometries for the four individuals. Numbers denote subjects. Column 1: Recovery time (RT); Column 2: Difference between diastolic RT and the RT of the nearest neighbor on systolic geometries; Column 3: $\mathrm{CC}_{\text {STT }}$ : correlation coefficient during STTsegment; Column 4: linear distance of each diastolic node to its nearest neighbor on the systolic geometry. LV: left ventricle. RV: right ventricle. A: Apex.

\section{Discussion}

In this paper, we quantitatively and systematically assessed the difference in inverse solution between systolic and diastolic geometries in ECGI in four subjects without structural heart disease, during sinus rhythm. Since the ventricular volume is highest during electrical systole and lowest during electrical diastole [5], it is assumed that the true geometries during the QRS-complex and the T-wave were diastolic and systolic, respectively. Overall, $\mathrm{r}_{\mathrm{AT}}, \mathrm{r}_{\mathrm{RT}}$, $\mathrm{CC}_{\mathrm{QRS}}$ and $\mathrm{CC}_{\mathrm{STT}}$ showed good correlation between both geometries and quantitative interpretation of electrical reconstructed ATs, RTs and electrograms of different geometries only differed little. This indicates that, at least quantitatively, epicardial potential maps and isochrones only differ slightly for the different phases of the cardiac cycle. Furthermore, differences in $\mathrm{AT}, \mathrm{RT}, \mathrm{CC}_{\mathrm{QRS}}$ and $\mathrm{CC}_{\text {STT }}$ scarcely correlated with linear movement or distance from apex, meaning that neither linear nor rotational movement contributes substantially to differences in epicardial potential maps and isochrones. These quantitative findings may further help in interpreting ECGI measurements, for the new and more accurate quantification of possible errors when interpreting repolarization. Furthermore, qualitative (and clinical) interpretation may still vary considerably due to regional differences in RTs that have large clinical implication but small quantitative effect (see Figure 2). For example, some pronounced areas of early or late repolarization in one geometry disappear in the other. However, these differences appeared to be arising mostly in areas of flat $\mathrm{T}$ waves (data not shown), which makes it hard to accurately determine RT of the local electrogram.

Our results are in contrast with our previous experimental study where we compared the movement of implanted electrodes to the reconstruction quality [2]. In that study, electrograms from epicardial electrodes which moved more, seemed to have lower correlation to inverse reconstruction at the corresponding ECGI node. However, that study was not designed to systematically study mechanical abnormalities and could analyze only one beat.

Even though the direct effect of assuming a static diastolic geometry in ECGI has not been studied yet in vivo, several studies have assessed the influence of varying geometries. Alday et al. have previously studied the effect of obtaining an inverse solution on the heart in an in silico study. [6] They reported that increasing the size of the heart for the inverse solution relative to the forward solution would give an increase of $\sim 2 \mathrm{~mm}$ in Euclidean distance error for estimating the focal excitation location. Even though we did not directly assess focal excitation location, the magnitude of the reconstruction error seems to be in line with their study. Tate et al. [7] quantitatively addressed the effect of segmentation differences in ECGI, and found 
that reconstruction differences on the epicardial surface mostly correlate with segmentation error, in contrast to our study, in which location differences between both geometries hardly correlated with reconstruction differences. The location of largest variance was also different: Tate et al. found that this was highest on the anterior surface, while this was the inferior surface in our study.[7] The difference in results might be due to the use of the spatiotemporal method in our study, the electrophysiological rhythm (RV and LV pacing produced the greatest effect in their study), the different outcome measures and the geometry of the heart itself.

As recently discussed in a consensus paper on ECGI validation [8], correlation coefficients do not always reflect clinical interpretation. Additionally, in some cases, it may be better to compare relative AT/RT differences instead of absolute values. However, we aimed for a global comparison by combining all three of these quantitative measures with quantitative maps, which are the most important metrics for clinical interpretation of the results.

The Tikhonov parameter was not fixed in this study. One might argue that the true differences in epicardial potential maps and isochrones would be reflected by fixing this parameter when calculating the inverse solution. However, since the Tikhonov parameter is directly dependent on the used geometry, we did not fix this parameter. Moreover, the ratio between the Tikhonov parameter between both geometries was $1.4 \pm 0.3$ on average, indicating that this had only a minor influence.

Even though we did assess the rotational movement of the heart, it was not measured directly. MRI may be more suitable to accurately track the different regions and nodes of the heart throughout the cardiac cycle. However, because of the very poor correlations between the distance from the apex and the inverse solution differences, we do not expect this to affect the corresponding results to a major extent.

Recommendations for future work would include using MRI to track tissue movement more accurately on a pointto-point basis, including a larger and more heterogeneous patient cohort and studying the effects on premature ventricular contractions.

Importantly, although it may appear that ECGI's reconstruction accuracy is not much influenced by the approximation of a mechanically non-moving heart, mechanical movement and electromechanical feedback are key elements in understanding arrhythmogenesis.

\section{Conclusion}

Overall, this first study investigating the influence of the use of a diastolic geometry during ECGI with conventional reconstruction methods shows that the inverse solution does not alter the overall quantitative interpretation of results notably. However, regional differences in recovery times, and hence the clinical interpretation may occur.

\section{Disclosure of conflicts of interest}

\author{
Matthijs Cluitmans is part-time employed by Philips \\ Research.
}

\section{References}

[1] M. J. Cluitmans, R. L. Peeters, R. L. Westra, and P. G. Volders, "Noninvasive reconstruction of cardiac electrical activity: update on current methods, applications and challenges," Neth Heart J, vol. 23, no. 6, pp. 301-11, Jun, 2015.

[2] M. J. M. Cluitmans, P. Bonizzi, J. M. H. Karel, M. Das, B. L. J. H. Kietselaer, M. M. J. de Jong, F. W. Prinzen, R. L. M. Peeters, R. L. Westra, and P. G. A. Volders, "In vivo validation of electrocardiographic imaging," JACC: Clinical Electrophysiology, vol. 3, pp. 232-242, 2017.

[3] B. Erem, D. H. Brooks, P. M. Van Dam, J. G. Stinstra, and R. S. MacLeod, "Spatiotemporal estimation of activation times of fractionated ecgs on complex heart surfaces." pp. 5884-5887.

[4] C. Goffinet, F. Chenot, A. Robert, A. C. Pouleur, J. B. le Polain de Waroux, D. Vancrayenest, O. Gerard, A. Pasquet, B. L. Gerber, and J. L. Vanoverschelde, "Assessment of subendocardial vs. subepicardial left ventricular rotation and twist using two-dimensional speckle tracking echocardiography: comparison with tagged cardiac magnetic resonance," Eur Heart J, vol. 30, no. 5, pp. 608-17, Mar, 2009.

[5] J. Baan, E. T. Van Der Velde, H. G. De Bruin, G. J. Smeenk, J. Koops, A. D. Van Dijk, D. Temmerman, J. Senden, and B. Buis, "Continuous measurement of left ventricular volume in animals and humans by conductance catheter," Circulation, vol. 70, no. 5, pp. 812-823, 1984.

[6] E. A. Perez Alday, D. G. Whittaker, A. Benson, and M. A. Colman, "Effects of heart rate and ventricular wall thickness on non-invasive mapping: an in silico study," Frontiers in Physiology, vol. 10, pp. 308, 2019.

[7] J. Tate, N. Zemzemi, W. Good, P. van Dam, D. Brooks, and R. MacLeod, "Effect of segmentation variation on ecg imaging," in 2018 Computing in Cardiology Conference, Maastricht, The Netherlands, 2018.

[8] M. Cluitmans, D. H. Brooks, R. MacLeod, O. Dossel, M. S. Guillem, P. M. van Dam, J. Svehlikova, B. He, J. Sapp, L. Wang, and L. Bear, "Validation and opportunities of electrocardiographic imaging: from technical achievements to clinical applications," Front Physiol, vol. 9, pp. 1305, 2018. 